\title{
Epidermolyse bulleuse héréditaire: difficultés de prise en charge en odontostomatologie.
}

\section{Bendoukha M1', Larbi Messaoudi S1, Dahmani K¹, Bouzouina F2}

1. Service de Médecine Dentaire : Hôpital Militaire Régional Universitaire Oran - Algérie

2. Service de Pathologie et Chirurgie Buccales - Algérie

Les Epidermolyses bulleuses congénitales ou héréditaires $(\mathrm{EBH})$ sont des génodermatoses rares, Leur prévalence estimée est de $8 / 1000000 \mathrm{cas} / \mathrm{hab} / \mathrm{an}$ et qui sont caractérisées par une fragilité cutanée et/ou muqueuse, localisée ou généralisée.

Cette fragilité anormale est due à des mutations de gènes codant pour des protéines impliquées dans la structure et la fonction de la jonction dermo-épidermique se traduisant cliniquement par la genèse de bulles ou d'érosions cutanées et parfois muqueuses de profondeur variable et de façon spontanée ou provoquée et au moindre frottement ou traumatisme. La sévérité de cette pathologie est très variable, allant des formes localisées permettant une vie normale aux formes généralisées handicapantes. [Fine J-Det al . Orphanet J Rare Dis 2010;28:5-12].

La classification basée sur le niveau histologique du clivage [Fine J-D et al .J Am Acad Dermatol2008;58:931—50] permet de distinguer les formes suivantes :

- Epidermolyses bulleuses simples (EBS)

- Epidermolyses bulleuses jonctionnelles (EBJ)

- Epidermolyses bulleuses dystrophiques (EBD) et

- Le syndrome de Kindler.

Dans les formes sévères généralisées, l'étendue des plaies, l'atteinte muqueuse, les cicatrisations à répétition sont sources de complications multiples : dénutrition, rétractions articulaires, carcinomes épidermoïdes cutanés. Le cas rapporté est celui d'une fillette de 12ans atteinte d'une EBH dystrophique sévère, nécessitant des hospitalisations fréquentes au service de pédiatrie et qui a été orientée par son médecin pour " des douleurs orales. ॥

L'examen clinique endo buccal s'est avéré difficile du fait de la limitation de l'ouverture buccale. Les secteurs molaires supérieurs et inférieurs présentaient un délabrement avancé. Un examen scannographique a été réalisé confirmant la généralisation et la gravité du processus carieux.

Dès le premier contact, on a pu évaluer la symptomatologie orale spécifique de la maladie et ses répercussions sur l'état général en particulier l'alimentation. Des difficultés ont été rencontrées lors de la prise en charge thérapeutique stomatologique.

Dans certains pays il est d'ailleurs préconisé l'extraction des secteurs postérieurs à titre préventif avant l'installation et l'aggravation de la microstomie. L'action prophylactique est donc nécessaire et doit être précoce.

Mots clés : dermatose héréditaire, bullose, microstomie, affection multidisciplinaire.

miriembendoukha@yahoo.fr

(C) The authors, published by EDP Sciences. This is an Open Access article distributed under the terms of the Creative Commons Attribution License 4.0 (http://creativecommons.org/licenses/by/4.0/). 